\title{
IDENTIFICATION OF SOURCES OF RESISTANCE IN SESAME AGAINST LEAF WEBBER AND CAPSULE BORER (ANTIGASTRA CATALAUNALIS DUP.)
}

\author{
AK PandaY*, MM Sundaria ${ }^{1}$, M Chandrasekaran ${ }^{2}$ ANd Rajani Bisen \\ All India Coordinated Research Project on Sesame and Niger, Jawaharlal Nehru Agricultural \\ University Campus, Jabalpur-482 004 (MP), India
}

Keywords: Sources of resistance, Leaf webber, Capsule borer, Sesame

\begin{abstract}
The 197 entries including two checks (SI-250 Resistance check and TC-25 Susceptible check) of sesame (Sesamum indicum L.) representing varied geographic and genetic diversity were tested at three diverse climatic locations of India viz., Jabalpur, Mandor and Vriddhachalam, against leaf webber and capsule. None of the screened entry was found to be free from infestation by leaf webber and capsule borer. The average plant, flower and capsule damage over the locations varied from 6.58 to $27.17,7.80$ to 23.71 and 3.33 to $15.43 \%$, respectively. At vegetative stage, the entries SI-0018-B (6.33\%) and IS-353-A (6.58\%) at flowering, the entry KMR-7 (7.80\%) and at capsule stage, the entries SI-0018-B (3.33\%), MT-67-25 (3.65\%) and RJS56-A $(3.80 \%)$ were recorded the lowest damage. Further, the response of promising entries under artificial pest load conditions over the locations showed that the entry SI-271-B was superior to others with respect to lowest plant and flower damage while at capsule stage, the entry NIC-8510-B was superior. Under artificial pest load condition, the entries SI-271-B, NIC-9839 and MT-67-25 showed the lowest damage whereas under natural condition, the entries IS-178-C and SP-3267 were superior to others with respect to lowest damage. The feeding preference studies showed that the entries SI-271-B, IS-178-C, MT-67-25 and S-OO-17-B were least preferred by the leaf webber and capsule borer and recorded the lowest leaf area damage.
\end{abstract}

\section{Introduction}

Sesame indicum L. origins in east Africa and India is one of the world's oldest oil seed crop grown mainly for its seeds that contain approximately 52 to $57 \%$ oil and $25 \%$ protein. Although it is grown in more than 55 countries, Asia contributes for more than 68 per cent area and 67 per cent production in the world. In the recent past, international demand and market for sesame has witnessed substantial growth. India ranks first in area (18.7 Lakhs) under sesame and earns Rs. 3000 crores through sesame export. Sesame is an excellent edible oil, food, biomedicine and health care, and all in one. The exceptional nutritional, medicinal, cosmetic and cooking qualities of sesame oil made it queen of oils. The seeds are rich in quality proteins and essential amino acids, especially methionine and tryptophan, which are essential for health. Sesame seed is a rich source of linoleic acid, vitamins, niacin and minerals including calcium and phosphorus. Sesame oil contains 85 per cent unsaturated fatty acids and is highly stable and has reducing effect on cholesterol and prevents coronary heart diseases. It is grown in all seasons of the year and being a short duration crop, fits well in to various cropping systems. In addition to India, substantial quantities of sesame are produced in Sudan, Myanmar and China. Gujarat, Rajasthan, Madhya Pradesh, Uttar Pradesh, West Bengal, Tamil Nadu, Maharashtra, and Andhra Pradesh are the major sesame producing states in India. However, productivity of sesame is low and fluctuating in India. Insect pests are one of the most important factors affecting the production of

*Author for correspondence: <pandeyjnkvv@gmail.com>, <pandey_bhu@rediffmail.com>. ${ }^{1}$ Agriculture Research Station, Mandor, Jodhpur-342304, India. ${ }^{2}$ ICAR-AICRP Centre for sesame, Tamil Nadu Agricultural University, Vriddhachalam-606 001, Tamil Nadu, India. 
sesame both in quality and quantity (Egonyu et al. 2005, Ahirwar et al. 2010). The pest attack tolls a heavy loss (25 to 90\%) in seed yield (Ahuja and Kalyan 2002). Though, sesame is attacked by a number of insect pests and mites, leaf webber and capsule borer (Antigastra catalaunalis) is the potential constraint to production from seedling stage to maturity (Choudhary et al. 1987, Selvanarayanan and Baskaran 1996). In a country like India, the production of sesame is already much below the expectation and therefore the damage due to Antigastra is undesirable. It is therefore, extremely important to devise means to reduce the extent of damage without adversely affecting the agro-ecosystem. Among the ecofriendly management measures, the use of resistant/tolerant varieties is one of the effective alternative which have no adverse effect on the ecosystem. So, resistant/tolerant variety is a right choice. Hence 197 entries of sesame against leaf webber and capsule borer were evaluated under natural and artificial pest load condition during Kharif season of the year 2011 and 2012.

\section{Materials and Methods}

Identification of sources of resistance in sesame against leaf webber and capsule borer was conducted at three diverse agro-climatic zones of India, JNKVV, Jabalpur (Madhya Pradesh), ARS, Mandor (Rajasthan) and RRS, Vriddhachalam (Tamil Nadu) during Kharif season of the year 2011 and 2012. One hundred ninety seven genotypes including resistant (SI-250) and susceptible check (TC-25) were taken as treatment to know their relative resistance/susceptibility against $A$. catalaunalis. The experiment was laid out in a rod-row design with single row of $5 \mathrm{~m}$ length, row to row and plant to plant spacing of $30 \mathrm{~cm}$ and $10 \mathrm{~cm}$, respectively. Recommended doses of fertilizers $(40 \mathrm{~N}+30 \mathrm{P}+20 \mathrm{~K} \mathrm{~kg} / \mathrm{ha})$ and other agronomic practices (except insecticides) were applied. Five plants of each genotype were selected randomly and tagged. Observations were recorded at vegetative, flowering and capsule stages by counting the number of damaged and total number of plant, flower and capsule per plant. The resistance/susceptibility for individual lines was judged on the basis of overall damage at all three stages of plant growth. Of which, ten promising entries were selected and screened under artificial pest load condition. Further for the confermation of resistance, the feeding preference studies of all the selected genotypes were also conducted.

$$
\text { Per cent leaf/flower/capsule damage }=\frac{\text { No. of infested leaf/flower/ capsule }}{\text { Total no. of leaf/flower/ capsule }} \times 100
$$

Susceptibility rating scale on the basis of overall damage at different stages (Vegetative, flowering and capsule stage) of plant growth

A. On the basis of plant and flower infestation
I. Infestation $<10$ per cent.
- Resistant
II. 10-20 per cent
- Moderately Resistant
III. 21-30 per cent
- Moderately susceptible
IV. 31-50 per cent
- Susceptible
V. above 50 per cent
- Highly susceptible
B On the basis of capsule damage
I. Infestation $<5$ per cent
- Resistant
II. 5-10 per cent
III. 11-15 per cent
IV. 16-25 per cent
V. above 25 per cent
- Moderately Resistant
- Moderately susceptible
- Susceptible
- Highly susceptible 


\section{Results and Discussion}

Results showed that none of the entry was free from the attack by the A. catalaunalis. However, significant differences were observed in the degree of infestation among the entries. At vegetative stage, the damage varied from 6.58 to $27.17 \%$, being lowest in the entry SI-0018-B and highest in the susceptible check (TC-25) followed by $26.38 \%$ in the entry EC-303454-A (Table 1). Flower damage was found to vary from 7.80 to 23.71 per cent while capsule damage was 3.33 to $15.43 \%$. At vegetative stage, the lowest damage was recorded in the entries SI-0018-B (6.33\%) and IS-353-A (6.58\%) while at flowering the entries KMR-7 (7.80), SI-1687 (8.38\%) and RJS-17 $(8.76 \%)$ were found to be superior to others (Table 1). At capsule stage, the lowest damage was recorded in the entries SI-0018-B (3.33\%), MT-67-25 (3.65) and RJS-56-A (3.80\%) (Table 1). Earlier Murli Bhaskaran and Thangavelu (1990) also reported resistance in terms of capsule damage in different germplasm lines of sesame which are more or less similar to present findings. On the basis of total damage at all the three stages of plant growth the screened genotypes which were grouped into different categories, showed that none of the them were resistant and moderately resistant. One hundred seventy four genotypes were categorized as susceptible with the range of 31 to 50\% damage. Three genotypes SI-1146, EC-303454-A and TC-25 were categorized as highly susceptible (>50\%) (Table 3). The present findings are in conformity with the results of Baskaran et al. (1994), Ahuja and Kalyan (2001), Manisegaran et al. (2001) and Singh (2002). They reported that the genotypes KMR-14 and TKG-22 were moderately resistant against $A$. catalaunalis.

Table 1. Response of genotypes of sesame against leaf webber/capsule borer at different locations of India (average of three locations and of two years data).

\begin{tabular}{llccccc}
\hline $\begin{array}{l}\text { Sl. } \\
\text { No. }\end{array}$ & Entry & $\begin{array}{c}\text { Plant } \\
\text { damage }(\%)\end{array}$ & $\begin{array}{c}\text { Flower } \\
\text { damage }(\%)\end{array}$ & $\begin{array}{c}\text { Capsule } \\
\text { damage }(\%)\end{array}$ & $\begin{array}{c}\text { Total damage } \\
(\%)\end{array}$ & Reaction \\
\hline 1 & GSM-22 & 10.98 & 13.03 & 4.75 & 28.76 & MS \\
2 & EC-310421 & 13.75 & 10.73 & 6.98 & 31.46 & $\mathrm{~S}$ \\
3 & IC-14093 & 16.85 & 11.53 & 6.72 & 35.10 & $\mathrm{~S}$ \\
4 & SI-2116 & 16.05 & 13.36 & 6.92 & 36.33 & $\mathrm{~S}$ \\
5 & GRT-83135 & 21.40 & 12.66 & 6.58 & 40.65 & $\mathrm{~S}$ \\
6 & MT-67-25 & 11.12 & 10.63 & 3.65 & 25.40 & $\mathrm{MS}$ \\
7 & NIC-16328 & 13.90 & 14.58 & 5.48 & 33.97 & $\mathrm{~S}$ \\
8 & NIC-8526 & 15.77 & 12.45 & 7.47 & 35.69 & $\mathrm{~S}$ \\
9 & NIC-16275 & 14.35 & 11.61 & 5.93 & 31.90 & $\mathrm{~S}$ \\
10 & SI-2973 & 15.48 & 13.21 & 6.73 & 35.43 & $\mathrm{~S}$ \\
11 & NIC-8984 & 14.02 & 14.65 & 6.43 & 35.10 & $\mathrm{~S}$ \\
12 & IS-52359-A & 12.10 & 16.95 & 6.12 & 35.17 & $\mathrm{~S}$ \\
13 & S-0429-A & 12.45 & 16.65 & 6.12 & 35.22 & $\mathrm{~S}$ \\
14 & GRT-00115-A & 21.26 & 15.20 & 7.33 & 43.79 & $\mathrm{~S}$ \\
15 & SI-1665 & 9.86 & 15.06 & 5.67 & 30.60 & $\mathrm{~S}$ \\
16 & OLT-61-A & 15.81 & 16.65 & 7.15 & 39.61 & $\mathrm{~S}$ \\
17 & IS-353-A & 6.58 & 15.45 & 5.90 & 27.93 & MS \\
18 & IS-413-A & 15.05 & 14.18 & 6.60 & 35.83 & $\mathrm{~S}$ \\
19 & NIC-17335-A & 12.47 & 18.46 & 6.13 & 37.07 & $\mathrm{~S}$ \\
20 & IS-280-A & 9.28 & 13.61 & 5.58 & 28.48 & $\mathrm{MS}$ \\
\hline & & & & & \\
\hline
\end{tabular}


Contd.

\begin{tabular}{|c|c|c|c|c|c|c|}
\hline $\begin{array}{l}\text { Sl. } \\
\text { No. }\end{array}$ & Entry & $\begin{array}{c}\text { Plant } \\
\text { damage }(\%)\end{array}$ & $\begin{array}{c}\text { Flower } \\
\text { damage }(\%)\end{array}$ & $\begin{array}{c}\text { Capsule } \\
\text { damage }(\%)\end{array}$ & $\begin{array}{c}\text { Total damage } \\
(\%)\end{array}$ & Reaction \\
\hline 21 & IS-296-A & 15.20 & 17.00 & 6.53 & 38.73 & $S$ \\
\hline 22 & IS-607-A & 13.32 & 13.01 & 6.01 & 32.34 & S \\
\hline 23 & SI-3178-I & 18.12 & 16.66 & 6.63 & 41.42 & $\mathrm{~S}$ \\
\hline 24 & RJS-56-A & 20.30 & 13.65 & 3.80 & 37.75 & S \\
\hline 25 & NIC-16095-A & 12.90 & 15.98 & 4.80 & 33.68 & S \\
\hline 26 & DSK-I-A & 17.18 & 16.88 & 5.17 & 39.23 & S \\
\hline 27 & IS-58-2-A & 20.53 & 10.40 & 7.25 & 38.18 & $\mathrm{~S}$ \\
\hline 28 & SI-318 & 17.52 & 17.80 & 5.20 & 40.52 & $\mathrm{~S}$ \\
\hline 29 & NIC-16401-A & 13.07 & 16.16 & 3.68 & 32.92 & S \\
\hline 30 & S-0062-A & 12.88 & 13.73 & 5.40 & 32.02 & S \\
\hline 31 & SI-1060 & 22.30 & 10.23 & 7.03 & 39.56 & S \\
\hline 32 & OLT-44 & 18.33 & 16.85 & 8.17 & 43.35 & S \\
\hline 33 & IS-481 & 20.17 & 16.75 & 4.05 & 40.97 & $\mathrm{~S}$ \\
\hline 34 & IS-425-C & 17.82 & 15.90 & 6.33 & 40.05 & $\mathrm{~S}$ \\
\hline 35 & IS-52 & 19.18 & 9.50 & 8.70 & 37.38 & S \\
\hline 36 & IS-552 & 18.70 & 11.75 & 9.63 & 40.08 & S \\
\hline 37 & SI-2670 & 15.92 & 16.08 & 6.38 & 38.38 & S \\
\hline 38 & SP-1162-B & 13.03 & 14.43 & 7.65 & 35.12 & $\mathrm{~S}$ \\
\hline 39 & IS-178-C & 7.90 & 10.00 & 3.90 & 21.80 & MS \\
\hline 40 & IS-56-1 & 17.53 & 14.35 & 6.43 & 38.32 & $\mathrm{~S}$ \\
\hline 41 & RJS-17 & 12.73 & 8.76 & 5.38 & 26.87 & MS \\
\hline 42 & IS-8480-B & 18.88 & 12.26 & 6.50 & 37.64 & $\mathrm{~S}$ \\
\hline 43 & IC-14160-I & 11.88 & 15.66 & 7.72 & 35.27 & S \\
\hline 44 & ES-110-C & 18.60 & 17.00 & 5.48 & 41.08 & S \\
\hline 45 & IS-607-1-84 & 23.80 & 9.86 & 9.70 & 43.37 & $\mathrm{~S}$ \\
\hline 46 & NIC-16236 & 17.93 & 12.06 & 9.10 & 39.09 & $\mathrm{~S}$ \\
\hline 47 & IS-722 & 19.38 & 15.63 & 6.30 & 41.32 & S \\
\hline 48 & ES-165-B & 16.97 & 16.20 & 6.93 & 40.10 & S \\
\hline 49 & SI-255-I & 14.68 & 15.21 & 5.97 & 35.87 & $\mathrm{~S}$ \\
\hline 50 & IS-104 & 9.43 & 13.28 & 4.78 & 27.50 & MS \\
\hline 51 & RJS-738-1-84 & 8.75 & 14.96 & 6.97 & 30.68 & $\mathrm{~S}$ \\
\hline 52 & IS 319-1 & 13.08 & 12.53 & 6.62 & 32.23 & S \\
\hline 53 & SI-3100 & 19.05 & 11.90 & 6.20 & 37.15 & $\mathrm{~S}$ \\
\hline 54 & IS-1848 & 10.25 & 13.85 & 5.80 & 29.90 & MS \\
\hline 55 & SI-1667-2 & 12.28 & 12.36 & 7.55 & 32.20 & $\mathrm{~S}$ \\
\hline 56 & IS-17-1 & 13.15 & 12.65 & 5.93 & 31.73 & $S$ \\
\hline 57 & ES-234-1-84 & 22.18 & 15.15 & 11.00 & 48.33 & S \\
\hline 58 & NIC-8252 & 14.43 & 15.26 & 8.90 & 38.60 & S \\
\hline 59 & ES-35-B & 18.95 & 18.55 & 6.42 & 43.92 & S \\
\hline 60 & SI-789 & 10.13 & 16.01 & 6.65 & 32.80 & $\mathrm{~S}$ \\
\hline
\end{tabular}


Contd.

\begin{tabular}{|c|c|c|c|c|c|c|}
\hline $\begin{array}{l}\text { Sl. } \\
\text { No. }\end{array}$ & Entry & $\begin{array}{c}\text { Plant } \\
\text { damage }(\%)\end{array}$ & $\begin{array}{c}\text { Flower } \\
\text { damage }(\%)\end{array}$ & $\begin{array}{c}\text { Capsule } \\
\text { damage }(\%)\end{array}$ & $\begin{array}{c}\text { Total damage } \\
(\%)\end{array}$ & Reaction \\
\hline 61 & S-0025 & 17.68 & 13.70 & 6.53 & 37.92 & $S$ \\
\hline 62 & IS-250 & 17.32 & 15.30 & 7.72 & 40.33 & $\mathrm{~S}$ \\
\hline 63 & NIC-8510-B & 13.90 & 10.35 & 4.78 & 29.03 & MS \\
\hline 64 & ES 72-C-B & 16.75 & 8.88 & 5.48 & 31.11 & $\mathrm{~S}$ \\
\hline 65 & IS-722-I & 11.75 & 15.18 & 13.22 & 40.15 & S \\
\hline 66 & IS-3051 & 12.53 & 17.50 & 13.40 & 43.43 & $S$ \\
\hline 67 & IS-191 & 13.57 & 14.63 & 15.07 & 43.27 & $S$ \\
\hline 68 & S-0374-A & 17.63 & 18.01 & 10.83 & 46.48 & $\mathrm{~S}$ \\
\hline 69 & KMR-54 & 18.70 & 12.98 & 9.83 & 41.51 & $\mathrm{~S}$ \\
\hline 70 & NIC-8562 & 12.13 & 10.91 & 5.85 & 28.89 & MS \\
\hline 71 & SI-102 & 16.83 & 13.05 & 8.27 & 38.15 & $\mathrm{~S}$ \\
\hline 72 & NIC-8062 & 13.75 & 9.95 & 6.00 & 29.70 & MS \\
\hline 73 & SI-1881-A & 12.83 & 13.26 & 8.03 & 34.12 & $\mathrm{~S}$ \\
\hline 74 & SI -7818-B & 10.80 & 15.26 & 6.35 & 32.42 & $S$ \\
\hline 75 & IS-615 & 15.70 & 17.08 & 7.45 & 40.23 & S \\
\hline 76 & KMR-71 & 16.98 & 10.70 & 3.68 & 31.37 & S \\
\hline 77 & ES 127-B & 21.55 & 18.75 & 8.73 & 49.03 & $S$ \\
\hline 78 & NIC-16237 & 13.97 & 12.25 & 5.48 & 31.70 & S \\
\hline 79 & SI-2182 -B & 22.58 & 9.65 & 9.88 & 42.10 & $\mathrm{~S}$ \\
\hline 80 & ES-3196 & 12.60 & 14.48 & 7.10 & 34.18 & $\mathrm{~S}$ \\
\hline 81 & SI-75 & 12.30 & 12.76 & 5.58 & 30.65 & $\mathrm{~S}$ \\
\hline 82 & IS-65 & 19.45 & 15.20 & 5.95 & 40.60 & S \\
\hline 83 & IS-74 & 19.45 & 19.33 & 5.55 & 44.33 & S \\
\hline 84 & NIC-10645 & 12.62 & 15.75 & 7.15 & 35.52 & $\mathrm{~S}$ \\
\hline 85 & SP-3267 & 7.50 & 11.01 & 6.02 & 24.53 & MS \\
\hline 86 & RME-111 & 22.02 & 15.83 & 6.55 & 44.40 & $\mathrm{~S}$ \\
\hline 87 & SI-953-B & 15.38 & 18.23 & 6.58 & 40.20 & $\mathrm{~S}$ \\
\hline 88 & SI-0018-B & 6.33 & 11.93 & 3.33 & 21.60 & MS \\
\hline 89 & IC-204962 & 18.60 & 12.36 & 9.33 & 40.29 & $\mathrm{~S}$ \\
\hline 90 & IS-715-1-84-B & 18.73 & 12.73 & 9.90 & 41.36 & S \\
\hline 91 & EC-303417-B & 15.78 & 11.43 & 9.18 & 36.38 & $\mathrm{~S}$ \\
\hline 92 & IS-152 & 14.42 & 15.38 & 11.83 & 41.63 & $\mathrm{~S}$ \\
\hline 93 & IS-1804-A & 19.35 & 20.13 & 8.20 & 47.68 & S \\
\hline 94 & NIC-16124-A & 14.50 & 14.01 & 8.87 & 37.38 & $S$ \\
\hline 95 & SI-1074-1 & 17.28 & 16.93 & 5.90 & 40.12 & $\mathrm{~S}$ \\
\hline 96 & EC-303454-A & 26.38 & 18.36 & 6.92 & 51.67 & HS \\
\hline 97 & NIC-16114-A & 17.17 & 17.46 & 7.38 & 42.02 & $\mathrm{~S}$ \\
\hline 98 & IC-204139 & 15.40 & 15.43 & 6.42 & 37.25 & $S$ \\
\hline 99 & SI-1188-I & 16.07 & 14.20 & 5.35 & 35.62 & $\mathrm{~S}$ \\
\hline 100 & IC-43177-A & 20.62 & 18.56 & 7.28 & 46.47 & $\mathrm{~S}$ \\
\hline
\end{tabular}


Contd.

\begin{tabular}{|c|c|c|c|c|c|c|}
\hline $\begin{array}{l}\text { Sl. } \\
\text { No. }\end{array}$ & Entry & $\begin{array}{c}\text { Plant } \\
\text { damage }(\%)\end{array}$ & $\begin{array}{c}\text { Flower } \\
\text { damage }(\%)\end{array}$ & $\begin{array}{c}\text { Capsule } \\
\text { damage }(\%)\end{array}$ & $\begin{array}{c}\text { Total damage } \\
(\%)\end{array}$ & Reaction \\
\hline 101 & SI-3279-1 & 20.43 & 12.06 & 9.20 & 41.69 & $S$ \\
\hline 102 & SI-0185 & 19.85 & 16.15 & 4.98 & 40.98 & S \\
\hline 103 & IC-205649 & 18.32 & 18.53 & 4.08 & 40.93 & $\mathrm{~S}$ \\
\hline 104 & NIC-9627 & 18.92 & 18.16 & 7.18 & 44.27 & S \\
\hline 105 & TC-14146-C & 18.95 & 19.91 & 6.17 & 45.03 & S \\
\hline 106 & IC-1025-A & 15.02 & 20.30 & 7.47 & 42.78 & S \\
\hline 107 & NIC-16227-A & 17.48 & 16.93 & 7.37 & 41.78 & S \\
\hline 108 & NIC-8224-A & 18.10 & 11.90 & 8.03 & 38.03 & S \\
\hline 109 & SI-3315-6-I & 14.63 & 16.30 & 8.73 & 39.67 & S \\
\hline 110 & GRT-8330-B & 14.30 & 16.33 & 6.87 & 37.50 & S \\
\hline 111 & EC-303441-B & 15.68 & 16.73 & 8.25 & 40.67 & S \\
\hline 112 & NIC-16278-A & 13.03 & 16.21 & 6.43 & 35.68 & S \\
\hline 113 & S-0403-A & 17.25 & 16.05 & 6.68 & 39.98 & $\mathrm{~S}$ \\
\hline 114 & NIC-8423-B & 12.95 & 14.41 & 6.32 & 33.68 & $\mathrm{~S}$ \\
\hline 115 & S-484 & 12.38 & 17.31 & 6.58 & 36.28 & $\mathrm{~S}$ \\
\hline 116 & KMR-74 & 12.20 & 16.05 & 5.85 & 34.10 & S \\
\hline 117 & IC-204550 & 15.17 & 16.30 & 7.53 & 39.00 & S \\
\hline 118 & GRT-839-A & 15.63 & 16.91 & 9.27 & 41.82 & $\mathrm{~S}$ \\
\hline 119 & KMR-89 & 16.38 & 15.70 & 6.72 & 38.80 & $\mathrm{~S}$ \\
\hline 120 & NIC-7907 & 16.14 & 18.03 & 8.80 & 42.98 & $\mathrm{~S}$ \\
\hline 121 & NIC-8392 & 12.87 & 15.30 & 4.87 & 33.03 & S \\
\hline 122 & KMS-342 & 13.17 & 12.60 & 4.90 & 30.67 & $\mathrm{~S}$ \\
\hline 123 & KMS-349 & 18.28 & 15.48 & 5.90 & 39.67 & S \\
\hline 124 & NIC-8489 & 14.60 & 8.88 & 7.33 & 30.81 & $\mathrm{~S}$ \\
\hline 125 & KMR-28 & 19.15 & 11.28 & 5.83 & 36.26 & $\mathrm{~S}$ \\
\hline 126 & SI-271-B & 11.30 & 9.38 & 4.90 & 25.58 & MS \\
\hline 127 & NIC-9839 & 15.23 & 15.08 & 4.52 & 34.83 & $\mathrm{~S}$ \\
\hline 128 & IS-470-A & 19.20 & 12.38 & 7.98 & 39.56 & $\mathrm{~S}$ \\
\hline 129 & NIC-9627-I & 9.20 & 11.78 & 5.55 & 26.53 & MS \\
\hline 130 & SI-1451 & 14.58 & 14.45 & 5.18 & 34.22 & $\mathrm{~S}$ \\
\hline 131 & G-43 & 18.98 & 16.81 & 6.97 & 42.77 & $\mathrm{~S}$ \\
\hline 132 & BS-61 & 22.85 & 17.25 & 10.13 & 50.23 & HS \\
\hline 133 & G-37 & 10.53 & 19.63 & 5.78 & 35.95 & $\mathrm{~S}$ \\
\hline 134 & RJS-77 & 18.78 & 11.56 & 11.38 & 41.72 & S \\
\hline 135 & ES-75 & 18.78 & 11.21 & 8.45 & 38.44 & S \\
\hline 136 & G-3 & 11.05 & 11.86 & 10.28 & 33.19 & $S$ \\
\hline 137 & NIC-8463 & 11.70 & 17.78 & 15.43 & 44.92 & S \\
\hline 138 & NIC-3181 & 16.40 & 15.81 & 6.10 & 38.32 & S \\
\hline 139 & $\mathrm{G}-45$ & 16.57 & 11.25 & 11.35 & 39.17 & S \\
\hline 140 & EC-3340998 & 23.43 & 12.46 & 11.23 & 47.12 & $\mathrm{~S}$ \\
\hline
\end{tabular}


Contd.

\begin{tabular}{|c|c|c|c|c|c|c|}
\hline $\begin{array}{l}\text { Sl. } \\
\text { No. }\end{array}$ & Entry & $\begin{array}{c}\text { Plant } \\
\text { damage }(\%)\end{array}$ & $\begin{array}{c}\text { Flower } \\
\text { damage }(\%)\end{array}$ & $\begin{array}{c}\text { Capsule } \\
\text { damage }(\%)\end{array}$ & $\begin{array}{c}\text { Total damage } \\
(\%)\end{array}$ & Reaction \\
\hline 141 & EC-334999 & 19.15 & 12.16 & 10.08 & 41.39 & $\mathrm{~S}$ \\
\hline 142 & NIC-7905 & 17.08 & 10.10 & 7.23 & 34.40 & S \\
\hline 143 & EC-334985-1 & 21.25 & 10.58 & 8.53 & 40.36 & $\mathrm{~S}$ \\
\hline 144 & SI-1225 & 18.43 & 8.91 & 7.25 & 34.59 & S \\
\hline 145 & IS-728 & 17.98 & 9.55 & 8.80 & 36.33 & S \\
\hline 146 & EC-335010 & 20.43 & 11.95 & 10.18 & 42.55 & $\mathrm{~S}$ \\
\hline 147 & NIC-9839 & 14.98 & 9.26 & 4.78 & 29.02 & MS \\
\hline 148 & BS-490 & 20.50 & 13.15 & 8.08 & 41.73 & $\mathrm{~S}$ \\
\hline 149 & IC-14178 & 16.35 & 10.96 & 5.95 & 33.27 & S \\
\hline 150 & IC-132415 & 18.48 & 11.50 & 8.73 & 38.70 & S \\
\hline 151 & ES-43 & 15.53 & 13.56 & 10.52 & 39.62 & S \\
\hline 152 & IC-132415 & 18.88 & 17.75 & 8.57 & 45.20 & S \\
\hline 153 & S-0502 & 17.73 & 17.83 & 9.32 & 44.88 & S \\
\hline 154 & MS-4-275 & 18.62 & 17.16 & 8.13 & 43.92 & S \\
\hline 155 & NIC-8535 & 13.83 & 12.78 & 5.12 & 31.73 & S \\
\hline 156 & SI-253 & 22.78 & 12.48 & 7.80 & 43.06 & S \\
\hline 157 & SI-2192 & 19.25 & 13.06 & 11.03 & 43.34 & S \\
\hline 158 & IS-393-1 & 17.45 & 16.53 & 6.75 & 40.73 & $\mathrm{~S}$ \\
\hline 159 & IS-446-1-64 & 9.67 & 11.65 & 4.13 & 25.45 & MS \\
\hline 160 & IC-199443 & 16.68 & 14.26 & 6.12 & 37.07 & $\mathrm{~S}$ \\
\hline 161 & EC-334966 & 11.68 & 14.35 & 7.95 & 33.98 & S \\
\hline 162 & KMR-1 & 20.37 & 11.70 & 7.47 & 39.53 & $\mathrm{~S}$ \\
\hline 163 & IS-366 & 9.00 & 10.76 & 6.30 & 26.07 & MS \\
\hline 164 & SI-995 & 8.68 & 14.26 & 8.77 & 31.72 & $\mathrm{~S}$ \\
\hline 165 & EC-303440 & 12.37 & 13.33 & 4.73 & 30.43 & MS \\
\hline 166 & IS-723 & 13.80 & 11.30 & 7.80 & 32.90 & $\mathrm{~S}$ \\
\hline 167 & S-0140 & 13.92 & 10.25 & 5.33 & 29.50 & MS \\
\hline 168 & SI-2138-2 & 14.92 & 13.90 & 6.80 & 35.62 & $\mathrm{~S}$ \\
\hline 169 & G-25 & 18.18 & 11.90 & 9.60 & 39.68 & S \\
\hline 170 & G-14 & 13.28 & 13.93 & 8.80 & 36.01 & $S$ \\
\hline 171 & IS-451 & 11.75 & 17.76 & 4.42 & 33.93 & S \\
\hline 172 & S-0598 & 18.53 & 14.16 & 6.88 & 39.58 & S \\
\hline 173 & SI-1687 & 17.08 & 8.38 & 7.00 & 32.46 & S \\
\hline 174 & EC-178-2 & 22.20 & 12.31 & 9.08 & 43.59 & S \\
\hline 175 & EC-334950-1 & 21.73 & 11.36 & 7.07 & 40.16 & S \\
\hline 176 & SI-2174-1 & 20.98 & 11.01 & 7.95 & 39.95 & $S$ \\
\hline 177 & EC-334992 & 15.05 & 11.58 & 9.60 & 36.23 & S \\
\hline 178 & SI-7192 & 22.90 & 9.35 & 7.25 & 39.50 & S \\
\hline 179 & ES-1501 & 21.68 & 12.61 & 12.98 & 47.27 & $\mathrm{~S}$ \\
\hline 180 & SI-1146 & 26.15 & 12.15 & 12.35 & 50.65 & HS \\
\hline
\end{tabular}


Contd.

\begin{tabular}{llccccc}
\hline $\begin{array}{l}\text { Sl. } \\
\text { No. }\end{array}$ & Entry & $\begin{array}{c}\text { Plant } \\
\text { damage }(\%)\end{array}$ & $\begin{array}{c}\text { Flower } \\
\text { damage }(\%)\end{array}$ & $\begin{array}{c}\text { Capsule } \\
\text { damage }(\%)\end{array}$ & $\begin{array}{c}\text { Total damage } \\
(\%)\end{array}$ & Reaction \\
\hline 181 & SI-29973 & 18.83 & 13.25 & 8.03 & 40.10 & $\mathrm{~S}$ \\
182 & SI-3263 & 23.00 & 11.33 & 9.58 & 43.91 & $\mathrm{~S}$ \\
183 & KMR-19 & 23.05 & 13.86 & 9.78 & 46.69 & $\mathrm{~S}$ \\
184 & IS-56-A & 21.95 & 13.85 & 11.58 & 47.38 & $\mathrm{~S}$ \\
185 & KMR-7 & 18.25 & 7.80 & 5.78 & 31.83 & $\mathrm{~S}$ \\
186 & NIC-16278-A & 19.10 & 12.65 & 10.50 & 42.25 & $\mathrm{~S}$ \\
187 & IS-129 & 16.83 & 13.41 & 10.90 & 41.14 & $\mathrm{~S}$ \\
188 & G-47 & 20.18 & 13.66 & 9.00 & 42.84 & $\mathrm{~S}$ \\
189 & SI-3315-16 & 16.25 & 12.13 & 8.30 & 36.68 & $\mathrm{~S}$ \\
190 & ES-120-1-84-B & 19.95 & 14.35 & 6.13 & 40.43 & $\mathrm{~S}$ \\
191 & S-99-A & 20.13 & 14.26 & 11.35 & 45.74 & $\mathrm{~S}$ \\
192 & IS-449 & 17.83 & 11.68 & 10.30 & 39.81 & $\mathrm{~S}$ \\
193 & IS-156-3-84 & 19.67 & 15.68 & 10.83 & 46.18 & $\mathrm{~S}$ \\
194 & IC-30884 & 13.95 & 15.36 & 7.33 & 36.65 & $\mathrm{~S}$ \\
195 & IS-564 & 23.18 & 16.08 & 8.48 & 47.75 & $\mathrm{~S}$ \\
196 & SI-250 (RC) & 15.37 & 10.55 & 6.57 & 32.48 & $\mathrm{~S}$ \\
197 & TC-25 & 27.17 & 23.71 & 14.95 & 65.83 & HS \\
\hline
\end{tabular}

HS = Highly Susceptible, MS = Moderately Susceptible, $\mathrm{S}=$ Susceptible

Table 2. Screening of promising genotypes against Antigastra under artificial pest load condition in net house at Jabalpur, Mandor and Vriddhachalam.

\begin{tabular}{llccccc}
\hline $\begin{array}{l}\text { S1. } \\
\text { No. }\end{array}$ & Entry & $\begin{array}{c}\text { Per cent plant } \\
\text { infestation }\end{array}$ & $\begin{array}{c}\text { Per cent } \\
\text { flower damage }\end{array}$ & $\begin{array}{c}\text { Per cent capsule } \\
\text { damage }\end{array}$ & Total damage & Reaction \\
1. & MT-67-25 & 7.41 & 12.07 & 9.44 & 28.92 & MS \\
2. & IS-178-C & 18.57 & 12.94 & 8.15 & 39.66 & S \\
3. & RJS-17 & 34.48 & 12.87 & 10.63 & 57.98 & HS \\
4. & NIC-8510-B & 19.63 & 10.21 & 3.63 & 33.47 & S \\
5. & S-0018-B & 19.16 & 14.28 & 8.63 & 42.07 & S \\
6. & SI-271-B & 7.02 & 9.08 & 7.32 & 23.42 & MS \\
7. & SI-1451 & 21.05 & 11.94 & 12.95 & 45.94 & S \\
8. & NIC-9839 & 8.25 & 11.74 & 6.94 & 26.93 & MS \\
9. & SI-253 & 24.48 & 16.45 & 13.13 & 54.06 & HS \\
10. & OSC 366 & 27.43 & 12.88 & 16.36 & 56.67 & HS \\
11. & TC-25 & 39.57 & 23.10 & 15.64 & 78.31 & HS \\
12. & SI-250 & 20.72 & 12.62 & 6.98 & 40.31 & S \\
\hline
\end{tabular}

Among the screened entries, 10 promising entries, on the basis of their performance at different stages of plant growth (vegetative, flowering and capsule stages) were selected and further screened under artificial pest load conditions (Table 2). The screened entries were further categorized in to different categories on the basis of their performance at different stages of plant growth. The results showed that at vegetative stage, three entries, SI-271-B, MT-67-25 and NIC9839 were less than $10 \%$ plant damage and categorized as resistance whereas at flowering, the entry SI-271-B registered 9.08\% damage and at capsule stage the entry NIC-8510-B showed 


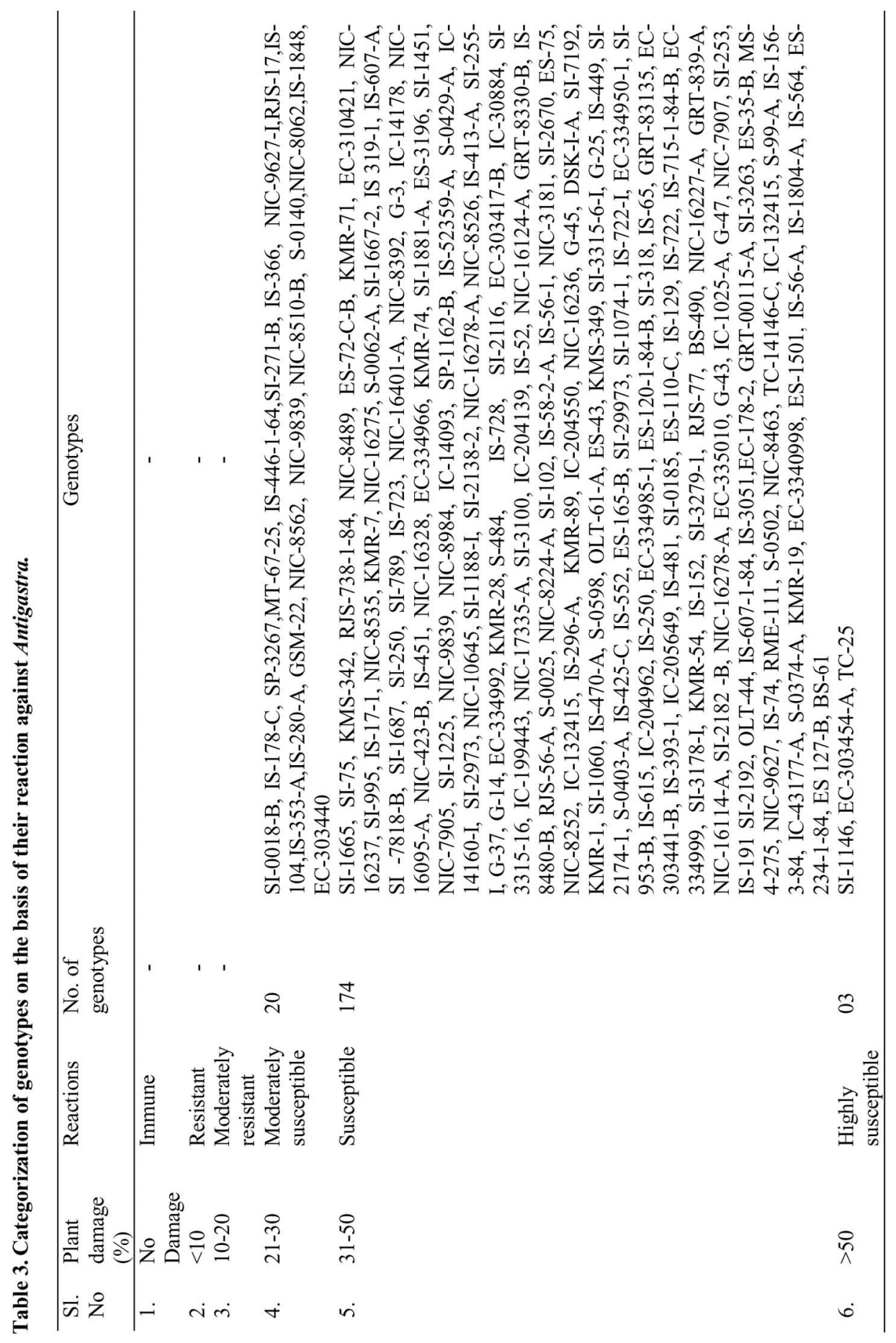


Table 4. Feeding preference studies in promising genotypes of sesame against Antigastra.

\begin{tabular}{|c|c|c|c|c|c|}
\hline \multirow{2}{*}{$\begin{array}{l}\text { Sl. } \\
\text { No. }\end{array}$} & \multirow[t]{2}{*}{ Entry } & \multicolumn{4}{|c|}{ Leaf damage $(\%)$} \\
\hline & & $\begin{array}{l}\text { No. third instar } \\
\text { larvae released }\end{array}$ & Mandor & Vriddchalam & Mean \\
\hline 1. & MT-67-25 & 10 & 4.08 (11.62) & $8.87(17.32)$ & 6.48 \\
\hline 2. & IS-178-C & 10 & $5.12(13.06)$ & $7.16(15.52)$ & 6.14 \\
\hline 3. & RJS-17 & 10 & $5.61(13.69)$ & $14.97(22.76)$ & 10.29 \\
\hline 4. & NIC-8510-B & 10 & $11.40(19.73)$ & $12.60(20.79)$ & 12.00 \\
\hline 5. & S-00-18-B & 10 & $5.21(13.16)$ & 10.57 (18.97) & 7.89 \\
\hline 6. & SI-271-B & 10 & 4.77 (12.59) & $6.91(15.24)$ & 5.84 \\
\hline 7. & SI-1451 & 10 & $8.04(16.45)$ & $15.41(23.11)$ & 11.73 \\
\hline 8. & NIC-9839 & 10 & $10.08(18.50)$ & $11.78(20.07)$ & 10.93 \\
\hline 9. & SI-253 & 10 & $8.85(17.31)$ & $14.07(22.03)$ & 11.46 \\
\hline 10. & OSC- 366 & 10 & $6.75(15.05)$ & $13.85(21.85)$ & 10.30 \\
\hline 11. & SI-250(RC) & 10 & $8.12(16.55)$ & $8.97(17.42)$ & 17.80 \\
\hline 12. & TC-25 (SC) & 10 & $7.07(15.41)$ & $27.47(31.61)$ & 8.02 \\
\hline \multicolumn{2}{|c|}{ SEM \pm} & & 0.44 & 0.94 & \\
\hline \multicolumn{2}{|c|}{$\mathrm{CD}$ at $5 \%$} & & 1.28 & 2.07 & \\
\hline \multicolumn{2}{|c|}{$\mathrm{CV} \%$} & & 4.98 & 7.95 & \\
\hline
\end{tabular}

$3.63 \%$ damage and categorized as tolerant. In short, the entry SI-271-B was found to be superior followed by MT-67-25. The results of feeding preference studies in Table 4 showed that the entries SI-271-B, IS-178-C and MT-67-25 were the least preferred entries whereas SI-250, NIC8510-B and SI-253 were highly preferred entries by A. catalaunalis. Germplasm lines have such inhibitory mechanism of resistance to Antigastra which can be used in transferring the resistance in to commercially viable varieties. Even partially resistant cultivars may also provide adequate control even with minimum usage of insecticides. It will help to prolong the useful commercial life of existing insecticides by discouraging the development of insecticide resistance strains of the insect.

\section{Acknowledgements}

Authors gratefully acknowledge the Project Coordinating Unit Sesame and Niger (ICAR), JNKVV, Jabalpur (M.P.) India for providing the financial support and necessary facilities for carrying out this study.

\section{References}

Ahirwar RM, Gupta MP and Banarjee S. 2010. Field efficacy of natural and indigenous products on sucking pests of sesame. Indian Journal of Natural Products and Resources 1(2): 221-226.

Ahuja DB and Kalyan RK 2001. Field screening of genotypes of sesame against leaf webber/capsule borer, Antigastra catalaunalis Dup., gallfly, Asphondylia sesami Felt and mite, Polyphagotarsonemus latus (Banks). Pest Manag. Econ Zool. 9(1): 409-412.

Ahuja DB and Kalyan RK 2002. Losses in seed yield due to insect pests in different varieties of sesame, Sesamum indicum L. Annals. Plant Soil Res. 4(1): 99-103.

Baskaran MRK, Ganesh SK and Thangavelu S 1994. Germplasm screening against sesame leaf roller and pod borer. Madras Agric. J. 81(11): 618-621. 
Choudhary R, Rai S and Singh KM. 1987. Economic injury level of the sesame leaf webber, Antigastra catalaunalis (Dup.) in Delhi. Indian J. Plant Prot. 15(2): 136-141.

Egonyu J P, Kyamanywa S, Anyanga W and Ssekabembe C K. 2005. Review of pests and diseases of sesame in Uganda. In African Crop Science Conference Proceedings. 7: 1411-1416.

Manisegaran S, Manimegalai N, Puspha J and Mohammed SEN 2001. Non- preference mechanism of resistance in sesame to shoot webber and capsule borer Antigastra catalaunalis (Dup.). Annals Plant Prot. Sci. 9(1): 123-124.

Murli Bhaskaran, RK and Thangavelu S 1990. Germplasm Screening against sesame leaf roller and pod borer, Sesame and Safflowr News Letter 5: 40-45.

Singh V 2002. Reaction of sesame genotypes to leaf webber and capsule borer Antigastra catalaunalis (Duponchel) (Lepidoptera: Pyraustidae). Sesame and Safflower Newslett. 17: 52-53.

Selvanarayanan V and Baskaran B 1996. Varietal response of sesame to the shoot webber and capsule borer, Antigastra catalaunalis Duponchel (Lepidoptera: Pyraustidae). Int. J. Pest Manag. 42(4): 335-336.

(Manuscript received on 3 July, 2018; revised on 2 November, 2020) 